Cinémas

Revue d'études cinématographiques

Journal of Film Studies

\title{
Action. Les passages narrativo-descriptifs du scénario
}

\section{Jacqueline Viswanathan}

Volume 2, numéro 1, automne 1991

Le Scénario

URI : https://id.erudit.org/iderudit/1001049ar

DOI : https://doi.org/10.7202/1001049ar

Aller au sommaire du numéro

Éditeur(s)

Cinémas

ISSN

1181-6945 (imprimé)

1705-6500 (numérique)

Découvrir la revue

Citer cet article

Viswanathan, J. (1991). Action. Les passages narrativo-descriptifs du scénario. Cinémas, 2(1), 7-26. https://doi.org/10.7202/1001049ar

\section{Résumé de l'article}

Il s'agit de mettre en question certaines des assertions les plus courantes des manuels d'écriture du scénario à propos des parties narrativo-descriptives. Que veut-on dire quand on conseille à l'aspirant scénariste de pratiquer une " écriture visuelle " ou une " écriture cinématographique "? L’analyse montre que, plutôt que des descriptions riches en détails visuels, les passages narratifs proposent une lecture des éléments signifiants essentiels du plan ou de la séquence, tels qu'interprétés et organisés par un spectateur hypothétique. 


\title{
Action. Les passages narrativo-descriptifs du scénario
}

\section{Jacqueline Viswanathan}

\begin{abstract}
RÉSUMÉ
Il s'agit de mettre en question certaines des assertions les plus courantes des manuels d'écriture du scénario à propos des parties narrativo-descriptives. Que veut-on dire quand on conseille à l'aspirant scénariste de pratiquer une «écriture visuelle» ou une «écriture cinématographique»? L'analyse montre que, plutôt que des descriptions riches en détails visuels, les passages narratifs proposent une lecture des éléments signifiants essentiels du plan ou de la séquence, tels qu'interprétés et organisés par un spectateur hypothétique.
\end{abstract}

\begin{abstract}
This article questions some common assertions found in handbooks about the writing of the action parts of a screenplay. What does "visual scriptwriting" really mean? An analysis of actual screenplays shows that, rather than descriptions with precise visual information, the narrative parts of a script focus on a reading of the most important signifying components of the shot or the sequence, as interpreted and organized by a hypothetical spectator.
\end{abstract}

Qui, parmi les critiques universitaires, ose juger un roman, une pièce ou un film suivant certaines normes préétablies? Qui, parmi les professeurs, prétend savoir enseigner comment s'écrit un bon poème? Les études littéraires et cinématographiques se veulent descriptives et analytiques plutôt que normatives ou pra- 
tiques. En revanche, la presque totalité des ouvrages publiés sur le scénario sont des manuels qui adoptent sans complexe une approche prescriptive ${ }^{1}$. On ne compte plus les livres s'intitulant: Comment écrire un scénario ou A Practical Manual of Screenplay Writing 2 . En raison de cette optique didactique, leur démarche suit presque toujours les différents stades d'écriture du scénario: invention de l'histoire (intrigue, personnages, situations); puis élaboration du récit (composition et découpage); enfin, rédaction du dialogue et parfois des directives scéniques et techniques. Leur parution remonte au tout début du cinéma et se poursuit de nos jours.

Bien que s'inspirant des multiples manuels américains du genre How to write a screenplay, il faut reconnaître que les auteurs français d'ouvrages récents, Michel Chion, Jean-Paul Torok, Anthony Cucca, Pierre Maillot et Jean-Claude Carrière, se gardent de présenter des recettes à succès garanti. À la différence des Américains, ils adoptent une approche plus analytique et exploitent des concepts de la critique actuelle, notamment de narratologie.

On aurait mauvaise grâce de condamner cette orientation pédagogique: le scénario est un texte qui répond à des besoins précis en fonction d'impératifs filmiques et commerciaux. Mais l'inconvénient d'une telle approche, c'est qu'elle peut parfois se limiter à des préceptes simplistes qui ne reflètent pas la pratique réelle et qu'elle encourage l'apprenti scénariste à se percevoir comme le docile exécutant d'un ensemble de règles. En mettant l'accent sur l'importance des recettes, les manuels contribuent peut-être aussi à faire sous-estimer la part artistique du scénariste, une injustice dont celui-ci a pâti depuis qu'existe le cinéma.

Je me propose ici de réexaminer certains lieux communs, se retrouvant de manuel en manuel (surtout dans le corpus américain) et qui me paraissent sujets à caution. Je ne chercherai pas à proposer de nouvelles règles d'écriture. Il me semble au contraire qu'il existe en fait une diversité de style parmi les scénaristes reconnus et que tout auteur devrait se sentir libre d'innover dans son écriture comme dans l'invention de l'histoire ou la composition du récit. Je me limiterai à l'analyse des parties narrativo-descriptives du scénario, autrement dites colonne «action» dans l'ancien format, ou encore directives scéniques dans le vocabulaire de théâtre (dorénavant désignées par l'abréviation ND dans mon texte). Que penser des conseils donnés à propos de la sélection de l'information ou du choix des procédés de narration et de style, en ce qui concerne ces passages? Est-ce que ces recommandations concordent avec certains 
exemples tirés de scénarios connus? Ces exemples, je les ai pris dans des découpages avant montage, publiés ou en manuscrits ${ }^{3}$. Quand je disposais de plusieurs «moutures», j'ai choisi de préférence la continuité dialoguée ou le découpage non technique, car je m'intéresse à la construction du monde imaginaire évoqué par les ND pour le film virtuel, plutôt qu'aux directives de tournage (cadrages, angles de prises de vues, etc.) qui visent sa fabrication.

$\mathrm{Si}$, suivant l'optique des manuels, le scénario est seulement perçu comme l'épure (blue print) d'un film à venir, il est normal de l'oublier ou même de s'en débarrasser après le tournage. Adoptant un autre point de vue, je voudrais au contraire consacrer la dernière partie de cet article à la question de l'édition du scénario. Il me semble en effet que la version des ND, choisie pour la publication peut influencer l'étude et l'interprétation des films, d'où l'importance d'une critique textuelle du scénario.

\section{Une écriture visuelle?}

«Il faut raconter en images», «... pratiquer une écriture visuelle», "À chaque idée, son plan ou presque. Le style écrit n'apporte rien au scénario et ne fait qu'alourdir le propos en cachant le plus important, la représentation visuelle. Il s'agit donc de figurer par des mots à la fois simples et précis, un découpage adapté4»: ce sont là les conseils qu'on adresse le plus fréquemment aux futurs scénaristes. À première vue, cela constitue de simples observations de bon sens, mais qui pourtant recèlent plusieurs présupposés contestables au sujet du langage et de ses rapports avec le visuel. L'auteur laisse entendre, par exemple, que le découpage par plans peut se superposer au «découpage» de la langue. On peut aussi se demander ce qu'il entend par «style écrit» et comment les mots peuvent «figurer» au lieu de signifier.

Un autre manuel propose un exemple qui nous permettra peut-être de mieux comprendre ce qu'est une «écriture visuelle». Tudor Eliad explique que la phrase «Le lendemain matin, X. se réveilla en pensant à elle» est inacceptable dans les ND d'un scénario parce que «non visuelle ${ }^{\text {»}}$. L'auteur propose de lui substituer: «Intérieur-jour. Soleil à travers les volets. $\mathrm{X}, \mathrm{mi}$ - éveillé, ouvre les yeux, puis jette de brefs regards autour de lui» (p. 58). On voit en effet que la deuxième phrase remplace une information temporelle et une information psychologique par des éléments «filmables»: soleil, regards. Mais le scénariste n'a pas, comme le dit Eliad, figuré le temporel et le psychologique par des éléments visuels. La deuxième phrase ne dit pas autrement, elle dit autre chose et laisse de côté les pensées de X. qui doivent 
pourtant constituer le message essentiel de ce plan. Un exemple tiré d'un scénario authentique, Les Enfants du Paradis de Jacques Prévert, révèle un processus inverse. Prévert écrit d'abord: «Frédéric ferme sa porte à clef»; puis il corrige: «Heureux d'être enfin seul, Frédéric ferme sa porte à clef en poussant un soupir de soulagement» (p. 28). Le scénariste a donc ajouté un trait psychologique non visuel en même temps qu'un second signifiant «visible» de ce sentiment: le «soupir de soulagement».

Les scénarios d'Ingmar Bergman sont eux aussi remarquables par la richesse de leurs notations psychologiques:

Enfin, Karen va vers le lit, se penche vers le visage de sa mère,
l'embrasse sur la joue. Puis, elle reste là, debout, immobile, très
longtemps et la regarde; elle a l'impression que la paupière de la
morte se contracte, que la poitrine se soulève pour respirer, mais
c'est une illusion - le jeu mystérieux de la mort avec l'esprit des
vivants (p. 112).

La dernière partie de cette citation décrit une illusion du personnage qui ne sera sûrement pas «visible» dans les images du film mais qu'un spectateur compatissant («spectateur idéal») pourrait imaginer. Il faut noter à quel point les scénarios de Bergman et ceux d'Antonioni, par exemple, diffèrent du modèle des manuels. On sent ici que les réalisateurs s'efforcent avant tout de communiquer un monde imaginaire, de donner vie et profondeur à leurs personnages pour leurs collaborateurs. Les ND de ces scénarios s'élaborent autour d'actions, de situations concrètes, mais elles les dépassent toujours par leur contenu émotif.

Il n'est donc pas nécessaire d'enjoindre aux futurs scénaristes d'exclure de leurs textes tout énoncé psychologique. Le scénario peut formuler les composantes essentielles du monde fictif construit par le spectateur à partir des plans du film. Ce monde inclut temporalité, causalité, continuité, tous concepts «non visibles» mais essentiels à la construction de l'intrigue et des personnages. Remarque banale pour la critique cinématographique mais qui ne paraît pas encore acceptée par certains professeurs de scénarió.

En fait, les passages narrativo-descriptifs du scénario ne sont pas riches en détails visuels: formes ou couleurs, par exemple. Ils ne tentent pas vraiment de décrire les images du film. Comment le pourraient-ils? Dans leur concision, ils proposent une lecture sémiotique de la séquence, comme le montre bien la première didascalie du scénario des Portes tournantes: 
Un violoniste noir d'environ 70 ans entouré de musiciens du même âge, en train de jouer un blues langoureux: "YOU DON'T KILL A PIANO PLAYER". On est au Foxy's, un club de Jazz qui a connu ses heures de gloire dans les années quarante. Aujourd'hui, il n'y a que des vieux. Ils sont tous noirs. Seule la pianiste est blanche. C'est Céleste. Elle a environ 75 ans, les cheveux blancs remontés en chignon. Son doigté souple, malgré l'âge, lui crée certaines difficultés. De temps à autre, elle jette un regard complice au violoniste (p. 2).

Il ne s'agit pas d'un texte à forte teneur visuelle. En revanche, ce court passage se caractérise par une sélection systématique des informations. Celles-ci peuvent se regrouper suivant trois isotopies: 1) la vieillesse: «70 ans, 75 ans, cheveux blancs en chignon, club qui a connu ses heures de gloire. Aujourd'hui, il n'y a que des vieux.» 2) la race: «ils sont tous noirs; seule, elle est blanche.» Ces deux isotopies impliquent des oppositions: aujourd'hui/hier; noir/blanc que la troisième permet de réconcilier: 3) la musique, liée à l'amour: «blues langoureux, doigté souple malgré l'âge, regard complice entre le violoniste noir et la pianiste blanche.» Cette brève description constitue l'épure du système signifiant de la première séquence. Le commentaire en voix «off» de Céleste va d'ailleurs guider le spectateur vers la «bonne» interprétation:

CÉLESTE, voix «off». - Tous les soirs, nous faisons de la musique au Foxy's, un club de Jazz qui a connu ses heures de gloire il y a longtemps. Pour nous parler, nous avons une langue qui n'est pas celle des autres. Les mots sont là bien sûr, mais l'onde qui nous berce est celle de la musique. Il m'arrive de voir l'émotion quitter son corps et venir tranquillement vers le mien. Les gens qui nous entourent ne comprennent pas. Ils sont dépassés par ce langage qu'ils n'essayent même plus d'expliquer (p. 3).

S'il y a redondance évidente entre la bande sonore et les ND, on remarquera aussi que celles-ci mettent en relief les oppositions d'âge et de race que le commentaire laisse pudiquement implicites mais qui ne manqueront pas de frapper le spectateur. La description également laconique du décor: «... au Foxy's, un club de Jazz qui a connu ses heures de gloire il y a longtemps» montre bien le peu d'importance de la visualité. Dans le scénario, il s'agit de caractériser le signe culturel plutôt qu'un référent particulier. Ceci vaudra notamment pour les lieux ou les vêtements: «une chambre dans le genre royaume des jouets", «une chemise à palmiers bleu pâle très Floride 1960». Cette formulation caractéristique des ND ne s'explique pas seulement, comme le disent les manuels, par des exigences de concision. Les «clichés» si fréquents dans les passages narrativo-descriptifs 
renvoient à des signifiés culturels qui peuvent être figurés par une multitude de signifiants possibles et comme camouflés par la richesse de l'image. Les scénarios regorgent de phrases aussi banales que: «Un amour attendri erre dans les yeux de la jeune femme» (manuscrit du scénario de J'accuse d'Abel Gance). Le monde profilmique et la séquence filmée enrichiront, nuanceront cette «lecture» emblématique de l'image.

Les manuels conseillent aux futurs scénaristes d'«écrire sous la dictée de l'image», en imaginant une sorte de film intérieur. Il faudrait ajouter que, en plus de la description de l'action, de nombreux scénarios évoquent les rapports entre divers plans ou séquences, qui correspondent aux opérations mentales nécessaires à la construction du récit, comme le montre cet autre extrait des Portes tournantes:

\section{INTÉRIEUR. JOUR. CHAMBRE D'ANTOINE}

Cette pièce fait contraste avec le studio de Blaudelle. On donne ici dans le genre «royaume des jouets».

Sur le mur, au-dessus du lit, une grande photo: on comprendra plus tard que c'est Lauda, la mère de l'enfant.

On retrouve Antoine, à moitié déshabillé, en train d'enregistrer un message (p. 7).

Nous y avons souligné tous les énoncés qui font spécifiquement référence à l'activité structurante du spectateur. Les manuels reconnaissent l'importance du travail du scénariste au niveau du récit, quand il ordonne les séquences et détermine ainsi le savoir et le non-savoir du public. Il faudrait aussi noter que les ND peuvent, elles aussi, évoquer une rhétorique du spectateur qui crée le suspense et la curiosité. Ainsi, ce passage du scénario de Citizen Kane:

INTERIOR. KANE'S BEDROOM. FAINT DAWN, 1940

A snowscene. An incredible one. Big impossible flakes of snow, a too picturesque farmhouse and snowman.

KANE, old voice. - Rosebud!

The camera pulls back showing the whole scene to be contained in one of those glassballs which are sold in novelty stores all over the world (p. 95).

Les ND du shooting script de Citizen Kane rendent compte de la réaction du spectateur d'abord hésitant entre la crédulité et l'illusion à cause de l'exagération de la scène, puis comprenant 
enfin le pourquoi de son artificialité. On voit aussi comment l'ordre de la description dans le passage suivant de Jésus de Montréal reflète la progression chez le spectateur, de la contemplation incrédule de cette scène surréaliste à la compréhension de la situation: il s'agit du tournage d'un film publicitaire:

Un étang de marbre, entouré de hauts buildings. Des jets d'eau jaillissent. Mireille, à peine vêtue de quelques chiffons diaphanes, s'avance vers nous. Elle marche sur l'eau, aérienne. Elle s'approche d'un homme très élégant, effleure sa joue d'un baiser furtif et disparaît.

\section{HOMME ÉLÉGANT}

«L'insaisissable légèreté de l'être.»

Il approche de son visage un flacon de parfum (p. 45).

Dans une conférence sur le scénario, Eisenstein insiste sur l'importance de la conceptualisation d'une rhétorique du spectateur dans la formulation des ND. Il compare deux versions du même scénario par des auteurs différents:

\section{1) The Banner}

A group of partisans approach a forest. Middle-aged farmers, old men, women, children. Fighting youths- - girls and boys.

Women on horseback. Men walking alongside them, leading cattle. And even though these are simple families fleeing their village captured by fascists, the detachment has the look of true partisans (p. 35).

\section{2) The Feast at Shirmunka}

A sky with billowy, peaceful clouds. Suddenly there is a whistle and the sound of shrapnel from the middle of the forest.

The sound of incomprehensible clanging and the murmur of excited voices. But the eye sees meadows and copses in the calm of summer stillness. Then we look down and see: something is going on in a sprawling border village. In front of the village soviet, weapons are being handed out to the inhabitants (p. 42).

Eisenstein déclare que le second passage est de loin supérieur au premier parce qu'il établit une sélection de plans propres à évoquer la curiosité du spectateur. Ce qu'il ne voit pas, ce qu'il ne comprend pas est un aspect essentiel de la programmation des images. Le style même du passage, le choix des adjectifs suggère les réactions du spectateur. 
À quel point le scénariste peut-il contrôler les images du film? À quel point peut-il rester maître de son œuvre? Par un choix d'informations purement visuelles, par la précision des directives techniques, comme semble le penser Syd Field?

But the screenplay writer as the prime mover can dictate. He can simply by writing in complete detail the necessary action and by writing in detail all dialogue and all character reactions and directions. He can dictate by timing all the action and the dialogue with a stop watch, to make certain that the length of the finished shot will conform with as little variation as possible to the length he feels necessary to the shot (p. 93).

On comprend l'anxiété du scénariste, auteur d'une œuvre incomplète; mais la cause est perdue. Il ne pourra jamais contrôler ainsi la réalisation. Il ne pourra jamais tout décrire du monde profilmique et de la cinématographie. On sait d'ailleurs que depuis les découpages techniques maniaquement précis dans la tradition de Thomas Ince ${ }^{7}$, même le scénario hollywoodien a abandonné ce format. En ce qui concerne cet aspect très limité du scénario qui nous intéresse ici, les ND, l'adéquation entre le texte et le film naîtra du pouvoir d'évocation de ces passages plutôt que de leur précision visuelle; les ND survivront en fonction de leur aptitude à suggérer le monde fictif, virtuel, construit par le spectateur. Étant bien entendu que le scénario se limite aux signes essentiels et qu'il ne peut exprimer qu'une infime partie de l'expérience du film vécue par le public de cinéma.

\section{Une écriture sans style?}

Le scénariste doit éviter à tout prix un «style littéraire» et ne doit surtout pas essayer d'imiter le romancier: c'est là une recommandation de la plupart des manuels de scénarios. L'examen des trois extraits suivants nous permettra peut-être de préciser ce qu'on entend par une absence de style:

\section{LE HALL D' ENTRÉE, 1}

Le hall d'entrée est un lieu relativement spacieux, presque parfaitement carré. Tout au fond à gauche, une porte mène vers les caves; au centre, la cage de l'ascenseur; sur la porte de fer forgé, un écriteau

\section{ARRÊT MOMENTANÉ DE L'ASCENSEUR}

a été accroché. Les murs sont laqués en vert clair, le sol est recouvert d'un tapis de corde d'une texture extrêmement serrée. 
Debout devant la loge, une femme est en train de lire la liste des habitants de l'immeuble; elle est vêtue d'un manteau de lin brun que ferme une broche pisciforme sertie d'alabandines (p. 115116).

La lumière baisse peu à peu. Sœur Julie a à peine le temps d'examiner une des cruches vides. Une cruche de verre comme celle dans lesquelles on achète du vinaigre. On peut encore voir l'étiquette verte: Une des cinquante-sept variétés Heinz.

La lumière disparaît tout à fait. On est plongé dans l'obscurité. L'alambic siffle et crépite à qui mieux mieux (p. 11).

Il fait noir. On ne voit que la petite lumière rouge de l'appareil cassette qui clignote. On devine Antoine étendu sur son lit.

On entend une chasse d'eau dans la pièce voisine. La porte de la chambre s'ouvre doucement et la tête d'Armande apparaît. Un rayon de lumière illumine le visage d'Antoine (p. 14).

Le lecteur aura-t-il deviné que les extraits (1) et (2) proviennent, le premier, du roman de George Pérec, La Vie mode d'emploi, le second, du roman d'Anne Hébert, Les Enfants du sabbat, et que seul le troisième vient d'un scénario: Les Portes tournantes de Jacques Savoie et Francis Mankiewicz? Il est certain que nous avons choisi ces passages «littéraires» à dessein (on pourrait presque dire qu'il s'agit de pastiches du scénario). Mais justement, cette possibilité d'imitation prouve qu'il existe un style «scénarique». Ce style n'est non «littéraire» que suivant une conception désuète de la littérarité, associée sans doute à une syntaxe complexe, une abondance de figures stylistiques, une charge émotive qui sont loin de caractériser la majorité des romans contemporains.

Ces trois extraits ont des caractéristiques communes: le même dépouillement - phrases nominales, style haché (peu de phrases complexes, de coordination entre les propositions), emploi du présent, absence de marques de l'énonciation (jamais de «je»), de signes d'affectivité ou d'expressivité. Le scénario renonce à certaines ressources du langage qui ne sont pas traduisibles dans le système de l'image. Ce que les romanciers empruntent au scénario, c'est cet effet de narration simultanée qui empêche tout recul narratif. Il ne s'agit pas d'une «absence de style» mais plutôt de traits stylistiques propres que le scénariste adopte quasi 
instinctivement en se plaçant dans la situation de discours propre au scénario.

Il est intéressant de noter que l'extrait (1) se «trahit», non par une différence de style, mais par la précision des détails visuels: couleur des murs, qualité du tapis, broche de la jeune femme. C'est que Pérec fait semblant de décrire un lieu qu'il aurait sous les yeux alors que le scénariste, qui se soucie peu de créer «une illusion de réalité», se contente d'évoquer un monde "virtuel», plus «générique». Le passage d'Anne Hébert pourrait sans aucun doute faire partie d'un scénario, par le rôle majeur qu'y joue la lumière et l'ombre, par la combinaison intéressante du son et de l'obscurité qui ressemble à l'effet évoqué dans l'extrait des Portes tournantes. La romancière utilise aussi comme signes d'ocularisation les syntagmes «On distingue», «on perçoit» qui correspondent à la perspective du spectateur, toujours présente dans le scénario.

Ici aussi, cependant, il faut se méfier des généralisations abusives. Éric Rohmer écrit les ND de certains de ses scénarios à la première personne. Un style plus «littéraire» (?) peut convenir à certains scénaristes. Ainsi, la seconde version du scénario préféré par Eisenstein (citée ci-dessus) est plus riche en signes d'affectivité. Les scénarios d'Ingmar Bergman regorgent de passages en purple prose où abondent les métaphores de toutes sortes, y compris les personnifications:

The enormous grandfather clock leans pensively against the wall, and the iron wood-stove turns away in rusty self-pity, while a bulky sofa in the heavy style of art nouveau is stretching in the floating, diffuse light... Curiously formed furniture monsters are asleep under white protective sheets, like snowed-in prehistoric animals dead long ago (p. 28).

Bergman se soucie peu de désobéir à toutes les règles d'écriture du scénario. Grâce aux ressources du langage, il préfère évoquer une atmosphère que le film traduira ensuite par son propre médium.

\section{Une écriture iconique?}

Jusqu'ici, nous avons envisagé les ND comme descriptions/narrations du contenu des plans ou des séquences du film. Les manuels de scénarios traitent également du rapport entre la façon de formuler ces passages et la forme même du film, les aspects de technique cinématographique. Il ne s'agit pas des directives techniques proprement dites (types de plan, par exemple). Celles-ci deviennent d'ailleurs de moins en moins fréquentes, même dans les scénarios hollywoodiens. Il est question d'un 
rapport mimétique entre l'organisation du discours narrativodescriptif du scénario et certains aspects formels du film. Ainsi, le scénariste pourrait pratiquer une écriture iconique qui mime certains aspects du cinéma: il s'agit, d'une part, du découpage et du cadrage (par un effet qu'on nomme souvent «cadrage ou découpage invisibles»); d'autre part, de la longueur et du rythme de succession des plans et des séquences.

Ainsi, dans cet extrait de Jésus de Montréal, les chiffres indiqués dans le texte correspondent aux plans du film:

BIBLIOTHÈQUE NATIONALE. INTÉRIEUR. JOUR.

Dessins de position de crucifiés dans un livre. (1)

Dans une encyclopédie moderne d'archéologie, dessin du crucifié de Jérusalem. (2)

(On est à la Bibliothèque nationale, à la mezzanine). (a)

Une bibliothécaire à l'allure sérieuse et pénétrée pousse un chariot chargé de livres jusqu'à la table. (3)

où Daniel travaille avec un gros crayon dans la bouche. (4)

Elle se penche vers lui, met des livres sur sa table (5) (p. 35).

Même si elle n'est pas rigoureuse, la correspondance entre découpage syntagmatique et découpage cinématographique est assez frappante. Seule, la phrase (a) ne concorde pas avec un plan du film mais pourrait facilement correspondre à un plan général. Il faut pourtant noter que d'autres ND ne se prêtent pas à ce type de superposition:

Elle se relève, vient au bord de la tranchée, avec une tablette de cire exactement semblable à celle que consultait Ponce Pilate. Elle la dépoussière avec les doigts (p. 66).

Ces propos correspondent à un seul plan dans le film.

Les conseils visant l'inscription d'un cadrage invisible dans le texte des ND préconisent également une correspondance analogique entre mode du discours (distance et focalisation) et le type et l'angle des plans. Dans le premier exemple de Jésus de Montréal cité ci-dessus, on voit bien que la sélection des informations suggère une certaine localisation dans l'espace, de l'instance d'énonciation par rapport à l'objet du discours: (1), (2) et (4) seraient des plans rapprochés; (a), un plan général puis (3) et (5) des plans moyens, ce qui correspond bien au cadrage du film. De plus, certaines ND peuvent suggérer un angle de prise de vue: «On aperçoit la statue de pierre, immense, devant le mur du sanctuaire» évoque une contre-plongée. Cette vision particu- 
lière peut être reliée à celle d'un personnage, Daniel («C'est la première station du Chemin de la croix. Daniel monte lentement les marches, regarde la statue»).

L'analyse du «point de vue personnel» est une des pierres de touche à la fois des critiques romanesque et filmique. On sait que l'analyse de la focalisation et, en particulier, de la rhétorique du «point de vue personnel» constitue aussi un domaine privilégié de l'étude comparée des récits romanesque et filmique. Nous n'allons pas revenir sur les travaux de F. Jost et F. Vanoye ${ }^{8}$. Ils ont bien montré la complexité des différences et des ressemblances entre les deux média, en ce qui concerne la modulation du point de vue. Il est curieux que les ND du scénario qui se situent justement «entre film et roman» ne soient pas plus explicites au sujet de l'ocularisation ${ }^{9}$. D'autre part, le scénario se distingue par l'inclusion explicite du point de vue du spectateur. De là, l'omniprésence des verbes de perception: «on voit», «on distingue»... Ainsi, dans ces extraits des Portes tournantes:

(Antoine) s'installe à la table et ouvre (le livre noir). En fouillant, il s'arrête de temps en temps, sur des bouts de lettres, des revues de mode et des vieux programmes. On voit la photo d'une femme avec un bébé sur les genoux.

On reste avec Blaudelle dans le studio... Il se dirige vers le téléphone. Pendant qu'il signale, on remarque une petite annonce accrochée au babillard (p. 15).

Y a-t-il une véritable analogie ${ }^{10}$ entre scénario et film, comme le laissent entendre les expressions «découpage et cadrage invisibles»? À notre avis, il s'agit seulement de métaphores et l'adjectif «invisible» révèle le désir du scénariste d'influencer subrepticement le metteur en scène.

Un autre aspect du scénario où pourrait se manifester une véritable écriture cinématographique, c'est la longueur et le rythme des plans et des séquences. Jean-Claude Carrière déclare, par exemple: «Il ne faut pas raconter longuement une action brève et brièvement une action longue. Le temps de lecture d'un scénario doit correspondre à peu près au temps de projection du film» (p. 79). Souvent, on conseille de compter environ une page de scénario par minute de film. Si la possibilité d'une équivalence temporelle entre dialogue écrit et dialogue parlé est déjà fort aléatoire, la synchronie entre temps de lecture des ND et temps de vision des séquences filmiques est encore bien plus problématique. Une analyse comparative du scénario et du film de Jésus de Montréal confirme ce qui tombe sous le sens ${ }^{11}$. Il n'y a pas de rapport entre la longueur des séquences dans le texte 
et leur durée dans le film. Ceci ne signifie évidemment pas que le scénariste n'influence pas le rythme filmique. Comme le montre bien Jean-Claude Carrière, les changements de lieux et l'alternance extérieur/intérieur ou jour/nuit, le type même d'actions racontées ou décrites impliquent un certain rythme, mais ils font partie du monde fictif et ne sont pas liés à une ressemblance entre la forme du texte et la forme du film.

Enfin, il me semble que les trois commandements cardinaux donnés à l'aspirant scénariste pour la rédaction des parties narrativo-descriptives - pratiquer une écriture visuelle, une écriture cinématographique, dans un style non littéraire - sont révélateurs d'une certaine difficulté d'être, d'une mauvaise conscience quant au statut du scénario par rapport au film. Afin de prendre ses distances vis-à-vis du roman, on poursuit le rêve impossible d'une écriture iconique qui se situe dans un rapport analogique avec le film.

Seuls les scénarimages ont ce rapport, mais ils ne sont pas pratique courante et constituent seulement un appoint. Il se peut cependant que la scénarisation à l'ordinateur, par images de synthèse, en facilite beaucoup l'utilisation. D'autre part, ceux qui souhaitent un scénario-partition, indiquant avec exactitude le rythme filmique, devraient recourir à un système symbolique semblable à la notation en musique. Dominique Zlatoff en a proposé un ${ }^{12}$. Jusqu'à présent cependant, ni le scénarimage ni le scénario-partition ne sont pratique courante, au stade du découpage non technique en tout cas. C'est que, par les ND, le scénario évoque les éléments et les structures essentielles du monde fictif tel que perçu et interprété par un spectateur, un monde distinct du film dans sa matérialité. Cette distinction entre le film, d'une part, et l'expérience filmique, objet de l'analyse, d'autre part, est un lieu commun de la critique cinématographique, mais elle n'a pas encore vraiment été acceptée par beaucoup de manuels de scénario. C'est la forme du contenu et non la forme de l'expression (suivant les termes de Hjelmslev) qui, dans le scénario, est structurée en fonction du médium filmique.

\section{Postérité du scénario}

À l'encontre des manuels qui ne voient dans le scénario qu'une épure du film à venir, un texte sans intérêt une fois le film terminé, nous voudrions maintenant aborder la question de la survie du scénario après le tournage (ou même sans tournage). Deux questions se posent: celle de la publication et celle du rôle du scénario comme instrument d'analyse, en particulier de l'influence que peuvent exercer les parties narrativo-descriptives sur l'interprétation d'un film. 


\section{Publications et éditions de scénarios}

La conception du scénario comme texte utilitaire a mené à la perte d'un grand nombre de manuscrits. C'est pourquoi il est tellement important que des collections spécialisées se constituent et continuent à s'enrichir dans les grandes cinémathèques, collections gérées par des bibliothécaires spécialistes du scénario. Ce sont les littéraires qui initient souvent la publication de scénarios et, par conséquent, ils privilégient les textes d'écrivains connus. Aux États-Unis, on a produit des éditions critiques très soignées de tous les scénarios de William Faulkner ${ }^{13}$ et de Scott Fitzgerald, y compris les scénarios qui n'ont jamais été tournés, et bien que cette partie de leur œuvre ne soit pas particulièrement admirée. En France, les scénarios de Sartre, Malraux, Giono, Vian ont aussi mérité une attention critique et la publication. Les scénaristes-réalisateurs voient souvent leurs textes publiés: Truffaut, Rohmer, Malle; au Québec, Denys Arcand. Les grands oubliés, ce sont les scénaristes qui n'ont pas d'autre titre de gloire. En ce qui concerne les normes d'édition, il règne encore une grande anarchie. Certains textes sont publiés sans qu'on indique s'il s'agit de découpages avant ou après montage, sans qu'on sache, par conséquent, qui a rédigé les ND: le scénariste ou d'autres, après visionnement du film.

En revanche, le scénario de La Belle et la Bête de Jean Cocteau, édité par Robert Hammond, est un modèle d'édition critique. Sans la persévérance de l'éditeur, le texte en aurait été irrémédiablement perdu. Cocteau avait égaré son tapuscrit. Le manuscrit original, découvert par Hammond, se trouve dans une collection privée suisse et n'est pas disponible. C'est par hasard que le critique américain en retrouve un exemplaire dactylographié chez Charles Spaak qui avait constitué une des rares collections de scénarios. Cette édition (avec traduction anglaise) du shooting script comprend aussi un découpage après montage avec notes. C'est grâce au travail de Hammond que l'on peut déterminer le caractère «apocryphe» d'une autre version parue en 1972. Les ND qui diffèrent considérablement du texte de Cocteau ont indubitablement été rédigées d'après le film. Or, nous l'avons vu, celles-ci représentent une véritable lecture des séquences et non une impossible description «objective». Une comparaison entre les deux textes montre, par exemple, une vision toute différente de la Bête: séduisant Grand Seigneur pour Cocteau, monstre loup-garou dans l'édition Grosset-Dunlap:

Cocteau: La Bête est un seigneur en grand costume de cour qui n'a d'une bête que la tête et les mains. La tête est celle d'un magnifique animal, sorte de lion aux yeux clairs. Son mufle 
miroite au soleil. Elle parle comme un être humain. Les mains sont des mains d'homme, velues et armées de griffes (p. 96).

\section{L'autre édition (même séquence mais ND toute différente):}

The Beast has the appearance of a werewolf with long fangs and grotesque features. His huge gnarled hands end in claws and like the rest of him are covered in thick matted fur (p. 209).

La description des costumes, par exemple celui de Belle, montre bien la différence de statut des deux textes. Il est clair que celles de l'édition Dunlap sont faites d'après les images du film alors que le scénario de Cocteau, comme tout shooting script, ne retient que les éléments de l'image essentiels à sa signification. Ainsi, Cocteau mentionne seulement le collier de Belle parce qu'il va jouer un rôle dans l'histoire en se transformant en corde. L'autre scénario nous donne des détails dignes d'une gravure de mode mais sans importance pour l'histoire:

(Cocteau): Belle porte une robe somptueuse et un collier de perles (p. 265).

(Grosset-Dunlap): Beauty looks like a princess. She is wearing a long white silk dress with full sleeves and a low-cut neckline. Her hair flows down her back in elaborate curls and on her head, she wears a jeweled coronet from which floats a translucent pearlstudded train. Her only piece of jewelry is a magnificent pearl necklace with a diamond clasp (p. 232-233).

On voit ici à quel point divers types de ND peuvent suggérer une vision toute différente de l'œuvre. Seule la version posttournage est une description des images du film. Le découpage avant montage en conçoit les structures signifiantes.

Ces différences sont particulièrement importantes parce que le scénario devient après coup un outil essentiel pour la critique cinématographique. Il est indispensable que toute édition de scénario spécifie clairement le statut du texte: à quel point a-t-il été modifié pour la publication, qui a rédigé les ND, avant ou après montage? Il est regrettable par exemple que les éditions des scénarios de Denys Arcand ou de Louis Malle ne donnent pas ces renseignements.

\section{Scénario et interprétation}

Toute analyse de film comporte des descriptions/narrations de plans ou de séquences. Les critiques sont évidemment conscients de la part de subjectivité et d'interprétation que comporte ce «discours sur l'image». Ainsi, ce commentaire de Jacques Aumont et Michel Marie dans L'Analyse des films: 


\begin{abstract}
Décrire une image - c'est à dire transposer en langage verbal les éléments d'information, de signification qu'elle contient, n'est pas une entreprise évidente, malgré son apparente simplicité. Beaucoup plus encore qu'une segmentation du film, la description détaillée des plans qui le composent présuppose un parti-pris analytique et interprétatif affirmé: en effet, il n'est pas question de décrire «objectivement» et exhaustivement tous les éléments présents dans une image et le choix auquel on se livre dans la description relève toujours en fin de compte, de la mise en œuvre d'une hypothèse de lecture explicite ou non (p. 49).
\end{abstract}

De nombreux critiques préfèrent ne pas se servir du découpage avant montage non seulement parce qu'il diffère du film terminé, mais aussi parce qu'ils préfèrent fonder leur interprétation sur leur propre lecture de l'image. Par exemple, dans son analyse minutieuse d'une séquence de Muriel, Marie-Claire Ropars prend soin de ne consulter le scénario de Jean Cayrol qu'après avoir fait sa propre transcription, description et analyse de la séquence.

Il est clair cependant que la continuité dialoguée ou le découpage avant montage constituent un texte de base pour certaines analyses. C'est le cas, par exemple, du chapitre sur Jésus de Montréal dans le livre de Heinz Weinmann ${ }^{14}$ qui fonde un des arguments principaux de son interprétation: le thème de la lutte entre Jésus et Mammon sur la première phrase des ND du scénario («la main prenant une liasse de billets dans la Bible»), séquence qui n'apparaît pas dans le film. Toutes les références au film d'Arcand renvoient aux pages du scénario. Certains commentaires reprennent directement la formulation des ND: «Commence alors une mêlée plutôt générale, une échauffourée comme dans un bar western" (même phrase dans le livre de Weinmann, p. 233). Un tel recours au scénario est d'ailleurs, à mon avis, tout à fait justifiable: la description de la séquence faite par Arcand lui-même (du moins, on le suppose) présentant autant d'intérêt que les versions après montage. On peut aussi exploiter la formulation des ND du shooting script pour éclairer les intentions de l'auteur, comme le fait Roger Viry-Babel, dans son article sur les films américains de Jean Renoir ${ }^{15}$, pour démontrer que, selon le scénariste/réalisateur, This Land is Mine n'est pas sensé se dérouler en France. Il est évident, dans ce cas, qu'il est essentiel de pouvoir identifier l'auteur des parties narrativo-descriptives.

\title{
Pour une critique du scénario
}

À côté de l'approche pédagogique adoptée par les manuels de scénario, il y a place pour une critique qui, au lieu de vouloir répondre à la question «comment écrire un bon scénario», se 
demande comment sont écrits les scénarios existants. Cette critique a déjà fait ses preuves avec le numéro spécial sur le scénario de la Revue de l'Université de Bruxelles, avec la revue spécialisée Les Cahiers du scénario, avec les thèses en voie de publication d'Isabelle Raynauld et Esther Pelletier ${ }^{16}$.

Il est facile de montrer que les expressions si rabâchées «écriture visuelle» ou «écriture cinématographique» posent des problèmes quand on veut leur donner une valeur autre que métaphorique. La question des rapports entre différents systèmes signifiants ainsi que celle du passage d'un système à l'autre sont extrêmement complexes et se situent au cœur même de la recherche sémiotique. Souhaitons qu'elles puissent nous apporter des réponses fructueuses pour la pratique du scénario.

\section{Simon Fraser University}

\section{NOTES}

1 Voir, par exemple, l'avant-propos de Tudor Eliad: «À quelles règles dramatiques, structurelles, psychologiques le scénario obéit-il? Comment écrire et vendre son scénario, guide pratique de l'aspirant scénariste, donne toutes les clés qui lui permettront de maîtriser ce nouveau genre si particulier.»

2 Voir notre bibliographie sommaire de manuels français et américains à la fin de cet article.

3 Puisque le nombre de scénarios publiés reste encore assez restreint, toute recherche dans ce domaine dépend de la disponibilité des manuscrits. C'est pourquoi je voudrais une fois de plus exprimer ma gratitude à Nicole Laurin de la Cinémathèque québécoise et à Dominique Brun du musée du cinéma HenriLanglois.

4 La dernière citation vient du «Dossier création», Caméra (3 février 1988). Elle ne fait que répéter le consensus des manuels renseignés dans la bibliographie de cet article.

5 Voir aussi, par exemple: «La vie psychologique sous toutes ses formes: dans un scénario, on n'a pas le droit de sentir, estimer, croire, penser, souffrir... On n'a pas non plus le droit d'avoir l'intention de... parce que les sentiments et toute la vie psychologique sont invisibles», Pierre Maillot, L'Écriture cinématographique (Paris: Méridiens Klincksieck, 1989) p. 216.

6 La richesse du contenu psychologique des ND dans certains textes se retrouve à travers toute l'histoire du scénario. Voir, par exemple, cet extrait du scénario de Sex de Gardner Sullivan (1920): "Overman comes through the door, he pauses a moment and stands there as he seeks to regain his balance. Naturally he cannot help being humiliated, but at the same time the calm manner in which Adrienne had disposed of him after he had proposed to her, appeals grimly to his sense of humor, and he exits with an ironic smile."

Les commentaires de Tom Stempel, qui cite ce passage, révèlent une conception originale du rôle du scénariste: "One can imagine what a sensitive director with a good actor could do with such a stage direction. In the film the shot 
begins with him outside the door, and the actor, William Conklin, gives a slight grimace and walks off. This is typical of the loss of emotional detail from script to film, not only in this film, but in other films Sullivan wrote." Tom Stempel, Framework, A History of Screenwriting in the American Film (New York: A Frederick Ungar Book, 1988) p. 42.

7 Voir Janet Staiger, «Blueprints for Feature Films: Hollywood's Continuity Scripts» in Tino Balio (ed.), The American Film Industry (Madison: The University of Wisconsin Press, 1985).

8 François Jost, L'Eil-caméra, Entre film et roman (Lyon: Presses Universitaires, 1987). Francis Vanoye, Récit écrit, récit filmique (Paris: Nathan, 1989).

9 Pour une caractérisation du point de vue narratif dans le scénario, voir: Isabelle Raynauld, «Le Point de vue dans le scénario», Protée (hiver-printemps 1988) p. 156-160; et Jacqueline Viswanathan, «Le Discours sur l'image», Semiotica, vol. 40, no 1/2 (1982) p. 27-43 et «Une histoire racontée en images», Études françaises $22 / 3$ (hiver 1987) p. 72-81.

10 Pierre Maillot utilise le terme «isomorphisme».

11 Jésus de Montréal: Séq. 1: 7p. = 73"; séq. 2: 1p.=138"; séq. 3: 2p. +10l. =90"; séq. 4: 1p. = 89"; séq. 5: 5 1/2p. = 166"; séq. 6: 1p. = 93".

12 Voir Dominique Zlatoff, «Pour une Forme scénarique véritablement cinématographique», "Autour du scénario», Revue de l'Université de Bruxelles $1 / 2(1986)$ p. $151-183$.

13 Bruce F. Kawin, Faulkner's MGM Screenplays (Knoxville: The University of Tennessee Press, 1982).

14 Heinz Weinmann, Cinéma de l'imaginaire québécois. De la petite Aurore à Jésus de Montréal (Montréal: L'Hexagone, 1990).

15 Voir Roger Viry-Babel, «Jean Renoir à Hollywood ou la recherche américaine d'une image française», Cinémas, vol. 1, no 1-2 (automne 1990) p.77. La citation est curieusement bilingue.

16 Isabelle Raynauld, Le Scénario de film comme texte. Histoire, théorie et lecture(s) du scénario de Georges Méliès à Marguerite Duras et Jean-Luc Godard (Paris: Université de Paris VII, 1990). Esther Pelletier, Écrire un scénario. Le scénario et l'industrie du cinéma québécois (Québec: Université Laval, 1990).

\section{OUVRAGES CITÉS}

The Beauty and the Beast. Translated by Carol Martin Sperry. New York: Grosset-Dunlap, 1972.

«Through a Glass darkly» in Three Films by Ingmar Bergman. New York: Grove Press, 1970.

Arcand, Denys. Jésus de Montréal. Montréal: Boréal, 1989.

Aumont, Jacques et Marie, Michel. L'Analyse des films. Paris: Nathan, 1988.

Bergman, Ingmar. Cris et Chuchotements. Paris: NRF/Gallimard, 1979. 
Carrière, Jean-Claude. «Réflexions d'un scénariste», "Autour du scénario», Revue de l'Université de Bruxelles 1/2 (1986).

Cocteau, Jean. La Belle et la Bête, texte inédit présenté par Robert M. Hammond. New York: New York University Press, 1970.

Eisenstein, Sergei M. On the Composition of the Short Fiction Scenario, translated by Alan Y Church. Calcutta: Seagull Books and Eisenstein Cine Club, 1984.

Eliad, Tudor. Comment écrire et vendre son scénario. Paris: Henri Veyrier, 1980.

Field, Sid. The Screenwriter's Workbook. New York: A Dell Trade Paperback, 1984.

Gance, Abel. J'accuse. Manuscrit du scénario conservé au musée du cinéma Henri-Langlois.

Hébert, Anne. Les Enfants du sabbat. Paris: Seuil, 1975.

Kaël, Pauline ed. The Citizen Kane Book. "The shooting Script" by Herman J. Mankiewicz and Orson Welles. Boston-Toronto: An Atlantic Monthly Press Book/Little, Brown \& Co., 1971.

Pérec, George. La Vie mode d'emploi. Paris: France Loisirs/Hachette, 1978.

Prévert, Jacques. Les Enfants du Paradis. Manuscrit conservé au Musée du cinéma Henri Langlois.

Savoie, Jacques et Mankiewicz, Francis. Les Portes tournantes. Scénario conservé à la Cinémathèque québécoise.

\section{BIBLIOGRAPHIE SOMMAIRE DE MANUELS DE SCÉNARIOS}

\section{Américains}

Armer, Alan. Writing the Screenplay. Belmont, California: Wadsworth Publishing Co., 1988.

Barker, E.F. Successful Photo-Play Writing. New York: Erie Publishing Co., 1914.

Bronfeld, Stewart. Writing for Film \& Television. New Jersey: Prentice-Hall, 1981.

Dmytryk, Edward. On Screenwritng. Boston-London: Focal Press, 1985.

Field, Sid. The Foundations of Screenwriting, A Step-by-step Guide. New York: Delta Book/Dell Publishing Co., 1979.

Herman, Lewis. A Practical Manual of Screenplaywriting. New York: New American Library, 1951.

Karetnikova, Inga. How Scripts are Made. Carbondale: University of Southern Illinois Press, 1990.

Marion, Frances. How to Sell and Write Film Stories. New York: CoviciFriede, 1937.

Packard, William. The Art of Screenwriting. New York: Paragon House Publishers, 1987.

Stempel, Tom. Screenwriting. San Diego: Barnes \& Co., 1982.

Vale, Eugene. The Technique of Screen \& Television Writing. New York: Simon \& Schuster, 1982.

Van Nostran, William. The Scriptwriter's Handbook. White Plains, New York: Knowledge Industry Publications, 1989. 


\section{Français}

Carrière, Jean-Claude et Bonitzer, Pascal. Exercice du scénario. Fondation européenne des métiers de l'image et du son, 1990.

Chion, Michel. Écrire un scénario. Paris: Cahiers du cinéma/INA, 1985.

Cucca, Antoine. L'Écriture du scénario. Paris: Dujarric, 1986.

Eliad, Tudor. Comment écrire et vendre son scénario. Paris: Henri Veyrier, 1980.

Maillot, Pierre. L'Écriture cinématographique. Paris: Méridiens Klincksieck, 1989.

Torok, Jean-Paul. Le Scénario. L'art d'écrire un scénario. Paris: ArtefactHenri Veyrier, 1986. 